\title{
Functional Equations Related to the Iteration of Functions
}

Report

Author(s):

Resch, R.; Stenger, F.; Waldvogel, Jörg

Publication date:

1996-10

Permanent link:

https://doi.org/10.3929/ethz-a-004284668

Rights / license:

In Copyright - Non-Commercial Use Permitted

Originally published in:

SAM Research Report 1996-14 


\title{
Functional Equations Related to the Iteration of Functions
}

\author{
R. Resch ${ }^{1}$, F. Stenger ${ }^{2}$ and J. Waldvogel
}

Research Report No. 96-14

October 1996

Seminar für Angewandte Mathematik

Eidgenössische Technische Hochschule

CH-8092 Zürich

Switzerland

\footnotetext{
${ }^{1}$ Filoli Information Systems, Palo Alto, CA 94304, USA

${ }^{2}$ University of Utah, Salt Lake City, USA
} 


\title{
Functional Equations Related to the Iteration of Functions
}

\author{
R. Resch ${ }^{1}$, F. Stenger ${ }^{2}$ and J. Waldvogel \\ Seminar für Angewandte Mathematik \\ Eidgenössische Technische Hochschule \\ CH-8092 Zürich \\ Switzerland
}

Research Report No. 96-14 October 1996

\begin{abstract}
Certain systems of functional equations related to the iteration of functions with a fixed point are considered. We construct smooth solutions in terms of expansions about the fixed point. In a particular example taken from an intuitive geometric situation the solution is obtained explicitly as a convergent Taylor series. Particular attention is given to the question of selecting distinguished solutions from an infinity of possible solutions. This classical topic is presented in a transparent way by consistently using compositional notation. The method described may be applied in similar situations, e.g. for handling iterations arising in discrete dynamical systems.
\end{abstract}

\footnotetext{
${ }^{1}$ Filoli Information Systems, Palo Alto, CA 94304, USA

${ }^{2}$ University of Utah, Salt Lake City, USA
} 


\section{Introduction}

Functional equations are relationships between values of a function at different arguments. In general they have larger sets of solutions than differential equations, which relate function values and derivatives at a single argument. Besides initial conditions additional requirements such as monotonicity are needed in order to select a distinguished particular solution.

The topic of functional equations has been active since the beginnings of calculus, e.g. with Euler's gamma function (see, e.g., [4]), up to the present day. A recent encyclopedia volume by Kuczma et.al. [9] contains an extensive bibliography of more than 800 references. The recent revival of the field is due to the connection between certain functional equations and the modern theory of dynamical systems.

In this paper we will begin with the intuitive geometrical situation drawn in Figure 1, below, which turns out to be intimately connected with the iteration of functions in one variable. In Section 2 the corresponding functional equations are solved by the classical methods of Schröder [10] and Abel [1]. Section 3 is devoted to the involutory case which is picked up in Section 4 by means of a particular example. In fact it is this example from three-dimensional geometry, being devised by R. Resch and publicised by F. Stenger [11] that gave the inspiration for this paper. In Sections 4 and 5 this example will be solved completely in terms of convergent power series.

Let $A(x), B(x)$ be two smooth monotonic functions defined on appropriate subintervals of $\mathbb{R}$, and consider their graphs as shown in Figure 1.

We discuss the problem of connecting the points of intersection of two polygons zigzagging between $A$ and $B$ by a simple smooth graph $G$. From Figure 1 we immediately obtain the conditions

$$
G(x)=A(y)
$$

$$
G(y)=B(x) .
$$

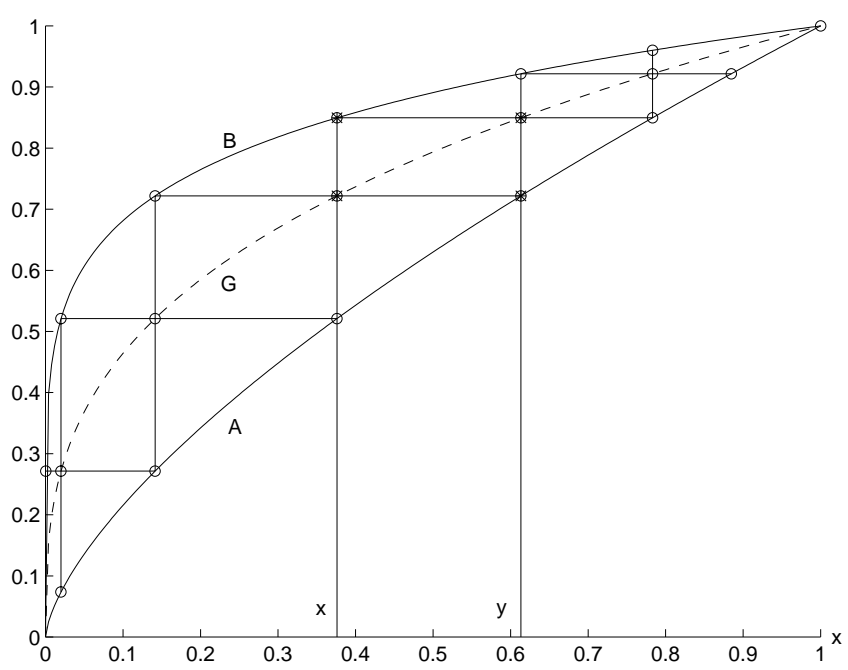

Fig. 1: Graphs of functions $A, B$ and $G$ 
Clearly, the solution is far from unique unless further requirements on $G$ are specified. Consider, e.g., the particular example $A(x)=a x^{\alpha}, B(x)=b x^{\beta}$ in $x \geq 0$ with $a, b, \alpha, \beta>0$. Assuming $G(x)=g \cdot x^{\gamma}$ shows that Equs. (1) and (2) are satisfied for every $x \geq 0$ and an appropriate function $y=Q(x)$ if we choose

$$
\gamma=\sqrt{\alpha \beta}, g=\left(a \sqrt{\beta} b^{\sqrt{\alpha}}\right)^{1 /(\sqrt{\alpha}+\sqrt{\beta})} .
$$

Here, this is the simplest possible solution. However, as will be seen later, there exist infinitely many more complicated solutions to the problem.

In this paper functions are denoted by capital characters, and composition will be denoted by juxtaposition, e.g.

$$
A(B(x))=(A B)(x) \text {. }
$$

Exponents denote functional iteration, such as $A^{n}(x)=A\left(A^{n-1}(x)\right), A^{0}(x)=x\left(A^{0}=I d\right.$, identity), and $A^{-1}$ accordingly denotes the inverse function of $A$.

\section{Functional Equations}

With the purpose of eliminating $y$ from the system (1), (2) we solve (1) for $y$, assuming that $A^{-1}$ exists:

$$
y=Q(x):=A^{-1}(G(x)), \text { or } \quad Q=A^{-1} G .
$$

Inserting this into (2) yields

$$
G A^{-1} G=B,
$$

and, with the abbreviation

$$
R:=A^{-1} B,
$$

the simple relation $Q^{2}=R$ is obtained. Hence the problem at hand amounts to taking the "compositional square root" $Q$ of the given function $R$; then

$$
G=A Q=A\left(A^{-1} B\right)^{1 / 2} \text {. }
$$

This is a particular case of so-called fractional iteration of a function which has a long history dating back at least to 1871 (E. Schröder, [11]). This early work was soon carried on by N. H. Abel [1], G. Königs [7], and E. Kasner [5], and the field has been active up to the present day. Besides the large bibliography in [9] a commented bibliography up to 1964 by Targonski [13] is mentioned. The question of uniqueness and regularity of growth

of fractional iterates was discussed, among many others, by Collatz [2], Davis [3], Kuczma [8], and Targonski [14].

We will use the methods of Schröder and Abel in order to construct particular solutions with various properties to the above problem. Using the idea of introducing appropriate 
"coordinates" [3], we introduce the formal conjugacy of the map $R$ with the shift map $S_{p}(x):=x+p$ ( $p$ is an appropriately chosen shift), by means of an Abel function or logarithm of iteration [1] $\Phi(x)$ for $R$ :

$$
\Phi R=S_{p} \Phi \text { or } \Phi(R(x))=p+\Phi(x) .
$$

If both $\Phi$ and $\Phi^{-1}$ exist, we have $R=\Phi^{-1} S_{p} \Phi$, and we immediately verify that

$$
Q=\Phi^{-1} S_{\frac{p}{2}} \Phi
$$

is a solution of Equ. (5).

Remark. The function $\Phi$ postulated here does not always exist; its existence depends on the behaviour of the rotation number of the map $R$. In the applications considered here, however, $\Phi$ exists based on convergent expansions in a fixed point of $R$.

In order to find more solutions consider the circle map $C$ with shift $p$, by definition a monotonic function

$$
x \longmapsto C(x):=x+P(x),
$$

where $P(x)=P(x+p)$ is a $p$-periodic function. Clearly, we have

$$
C S_{p}=S_{p} C
$$

Theorem 1 If $\Phi$ is an Abel function for $R$ with shift $p, \Phi R=S_{p} \Phi$, and if $C$ is a circle map with the same shift, the function $\widetilde{\Phi}=C \Phi$ is also an Abel function for $R$ with shift $p$.

Proof: $\Phi R=S_{p} \Phi \Longrightarrow C \Phi R=C S_{p} \Phi \Longrightarrow \widetilde{\Phi} R=S_{p} \tilde{\Phi}$.

Consequently, in Equ. (9) $\Phi(x)$ may be replaced by $\tilde{\Phi}(x)=\Phi(x)+P(\Phi(x))$, where $P$ is an arbitrary $p$-periodic function with the only restriction that $\widetilde{\Phi}^{-1}$ exists.

In the example of Section 1 we obtain

$$
R(x)=\left(\frac{b}{a} x^{\beta}\right)^{1 / \alpha}
$$

and, e.g.,

$$
\Phi(x)=\log \log (\mu x), \mu=\left(\frac{b}{a}\right)^{1 /(\beta-\alpha)}, p=\log \left(\frac{\beta}{\alpha}\right) .
$$

Choosing an arbitrary circle map $C$ with shift $p$, Equs (4), (9) yield $G=A \Phi^{-1} C^{-1} S_{\frac{p}{2}} C \Phi$ or

$$
G(x)=a\left(\frac{1}{\mu} \exp \exp \left(C^{-1}\left(\frac{1}{2} \log \frac{\beta}{\alpha}+C(\log \log (\mu x))\right)\right)\right)^{\alpha} .
$$

With $C=I d$ the simple solution $G(x)=g \cdot x^{\gamma}$ with $g, \gamma$ from $(3)$ is obtained. 


\section{Symmetry}

We now consider the case of involutions $A, B$ with $A^{2}=B^{2}=I d$. Then involutory solutions $G$ of (1), (2) may exist since $G^{2}=I d$ is compatible with $B^{2}=G A^{-1} G^{2} A^{-1} G$ $=I d$ (see Equ. (5)). We then have from (6) $R=A B, R^{-1}=B A$.

In order to construct an involutory solution we state the following theorem that holds without the symmetry condition on $A$ and $B$. Let $N(x)=-x$ be the "anti-identity"; we have $S_{p} N=N S_{-p}$.

Theorem 2 Let $\Phi$ be an Abel function for $R$ satisfying (8) or $\Phi A^{-1}=S_{p} \Phi B^{-1}$. Then the function $\tilde{\Phi}:=N \Phi A^{-1}$ satisfies $\tilde{\Phi} A=S_{p} \tilde{\Phi} B$.

Proof: $\Phi A^{-1}=S_{p} \Phi B^{-1} \Longrightarrow N S_{-p} \Phi A^{-1}=N \Phi B^{-1} \Longrightarrow S_{p} \tilde{\Phi}=\widetilde{\Phi} A B^{-1} \Longrightarrow S_{p} \tilde{\Phi} B=$ $\tilde{\Phi} A$.

Corollary. If $A$ and $B$ are involutions, $A^{-1}=A, B^{-1}=B$, and $\Phi$ satisfies $\Phi A=S_{p} \Phi B$, then $\tilde{\Phi}:=N \Phi A^{-1}$ satisfies the same equation, $\tilde{\Phi} A=S_{p} \tilde{\Phi} B$.

Next, we observe that any linear combination $w \Phi+\tilde{w} \tilde{\Phi}$ with $w+\tilde{w}=1$ satisfies Equ. (8). In particular, if we use $w=\widetilde{w}=\frac{1}{2}$ to define

$$
\Psi=\frac{1}{2}(\Phi+\tilde{\Phi}) \quad \text { or } \quad \Psi(x)=\frac{1}{2}\left[\Phi(x)-\Phi\left(A^{-1}(x)\right]\right.
$$

as our standard Abel function we have

\section{Theorem 3 The solution}

$$
G=A \Psi^{-1} S_{\frac{p}{2}} \Psi
$$

generated by the Abel function $\Psi$ is involutory.

Proof: We have $\Psi A=N \Psi$. Therefore

$$
G^{2}=A \Psi^{-1} S_{\frac{p}{2}} \Psi A \Psi^{-1} S_{\frac{p}{2}} \Psi=A \Psi^{-1} S_{\frac{p}{2}} N S_{\frac{p}{2}} \Psi=A \Psi^{-1} \Psi A=I d .
$$

The symmetry of the solution (14) may be displayed by writing (14) as $\Psi A^{-1} G=S_{\frac{p}{2}} \Psi$ or as

$$
\Phi\left(A^{-1}(G)\right)+\Phi\left(A^{-1}(x)\right)=p+\Phi(G)+\Phi(x) .
$$

This is an implicit equation of the curve $G(x)$ in the $(x, G)$ plane. Obviously, it is invariant if $x$ and $G$ are interchanged (hence involutory), and due to its construction it solves the problem (1), (2). $\Phi$ is an arbitrary Abel function for $R$ satisfying (8), and $p$ is the corresponding shift. More solutions are obtained by using $C \Phi$ instead of $\Phi$ in Equ. (15), where $C$ is a circle map with shift $p$. 
To conclude this section we derive an elegant parametrization of the curve defined by Equ. (15). With the definition

$$
D(x):=\frac{1}{p}\left(\Phi\left(A^{-1}(x)\right)-\Phi(x)\right)
$$

(15) becomes $D(G)+D(x)=1$. By introducing the parameter $t:=D(x)$, or $x=D^{-1}(t)$, where $D^{-1}$ is the inverse of $D$, we obtain the simple parametric form

$$
x=D^{-1}(t), \quad G=D^{-1}(1-t), \quad-\infty<t<\infty
$$

for the curve defined by Equ. (15).

\section{An Example}

In the $x y$-plane, consider two points: $A_{1}$ on the positive $x$-axis, and $A_{2}$ on the positive $y$-axis, such that the distance from $A_{1}$ to $A_{2}$ is always 1 . Thus, if $A_{1}=(x, 0)$, then $A_{2}=\left(0, \sqrt{1-x^{2}}\right)$.

Next, consider the "rod" $A_{1} A_{2}$ of unit length moving in the first octant of $x y z$-space, for $0 \leq z \leq 1$, in such a way that $A_{2}$ is always in the $y z$-plane $(x=0)$ and $A_{1}$ is always in the $x z$-plane $(y=0)$, and such that both $A_{1}$ and $A_{2}$ always have the same $z$-coordinate (see Figure 2). Two trajectories are of particular interest:

- The case when $A_{1}$ traverses the straight line trajectory $x=A_{1}(z)=1-z$, in which case $A_{2}$ traverses the circular path, $y=A_{2}(z)=\sqrt{1-(1-z)^{2}}$; and

- The case when $A_{1}$ traverses the circular trajectory $x=A_{1}=\sqrt{1-z^{2}}$, in which case $A_{2}$ traverses the straight line trajectory $y=A_{2}(z)=z$.

Let us now examine the family $\mathcal{F}$ of paths $x=G(z)$ of $A_{1}$, described as in the above paragraph, whose $x$-coordinate lies between the curves

$$
x=A(z):=1-z \text { and } x=B(z):=\sqrt{1-z^{2}}, \quad 0 \leq z \leq 1 .
$$

It is clear that any such trajectory will have a $y$-coordinate that lies between the trajectories $y=A_{2}(z)=\sqrt{1-(1-z)^{2}}$ and $y=A_{2}(z)=z$. Upon replacing $z$ by $1-z$ in the family of trajectories $G(z)$, we may ask: For what trajectories $G(z)$ do we have

$G(1-z)=A_{2}(z)$ ? Equivalently, since $A_{2}(z)=\sqrt{1-G^{2}(z)}$, for what functions $G$ defined on $[0,1]$, and such that $0 \leq G(z) \leq 1$ do we have

$$
G(1-z)=\sqrt{1-G^{2}(z)} ?
$$




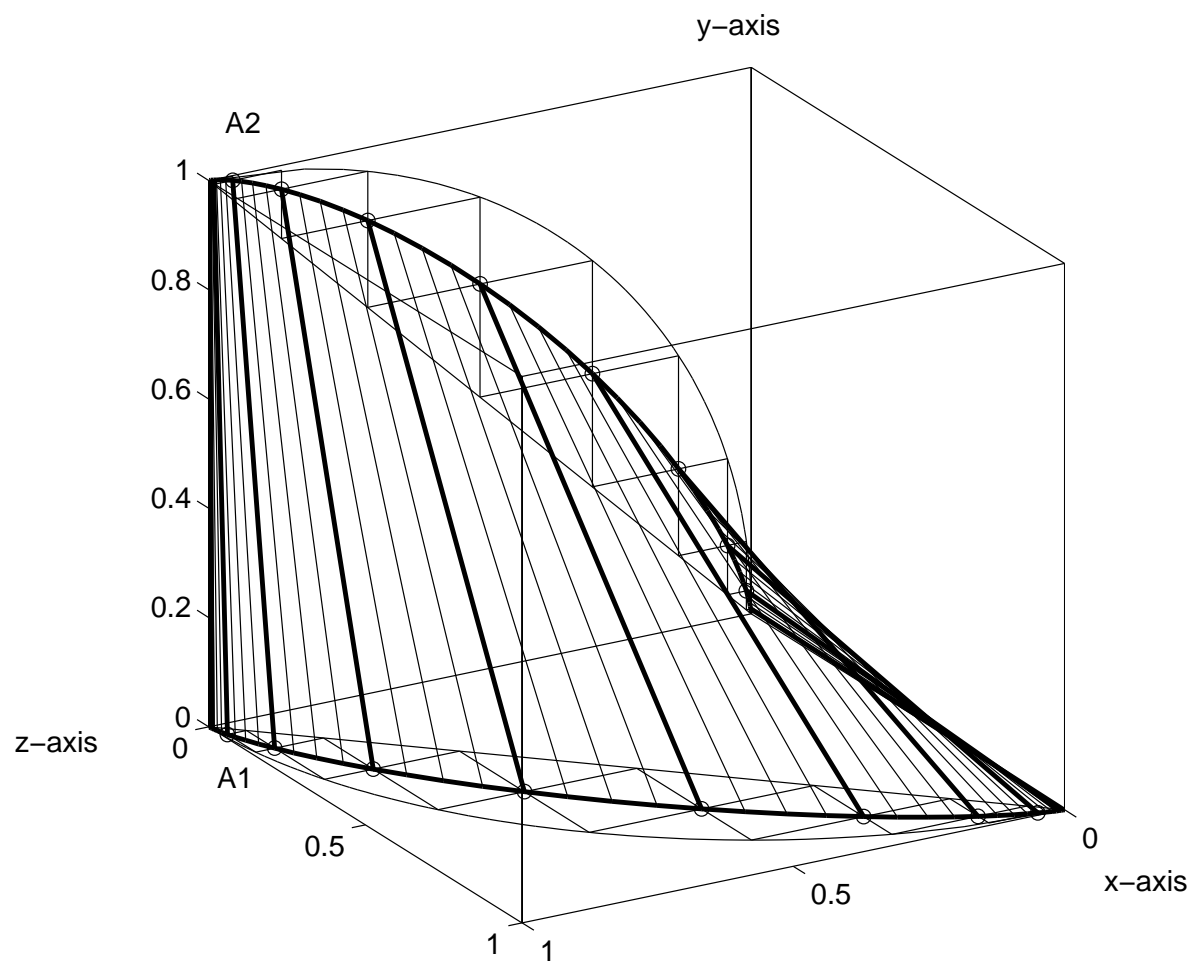

Fig. 2: The $\operatorname{rod} A_{1} A_{2}$ parallel to the $x y$-plane moving in such a way that the endpoints $A_{1}$ and $A_{2}$ traverse congruent paths

We notice that the functions $A$ and $B$ are involutory, i.e. on the interval $[0,1]$ they satisfy $A^{-1}(z)=A(z)$ and $B^{-1}(z)=B(z)$, and we furthermore restrict the family $\mathcal{F}$ of functions $G$ such that

$$
G^{-1}(z)=G(z)
$$

for all of the functions $G \in \mathcal{F}$. We may now ask:

- What are the properties of the family $\mathcal{F}$ ?

- In what sense is the family $\mathcal{F}$ described as above unique?

- If the family $\mathcal{F}$ consists of more than one member, what other properties reduce $\mathcal{F}$ to a single member $G$ ?

- Is it possible to construct such a $G \in \mathcal{F}$ ?

We shall consider these questions in turn, in what follows.

We first observe that due to (20), i.e. $G^{2}=I d$, all the symmetries of Section 3 apply. Furthermore, Equ. (19), which corresponds to $G A^{-1}=B G$ in the compositional notation of the previous sections, is a direct consequence of (5) and (20). Therefore the theory of 
Sections 2, 3 holds. Instead of Equ. (8) we use the equivalent form

$$
\Phi(1-z)=p+\Phi\left(\sqrt{1-z^{2}}\right)
$$

with the goal of choosing an appropriate value of $p$ and a simple solution $\Phi(z)$, e.g. a formal ascending series centered at $z=0$. It is suggested to adopt the ascending series solution $\Phi(z)$ to be defined below as the "best" solution of Equ. (21) in the sense that it shows the most regular growth as $z \rightarrow 0$. Other choices may be possible, though. Due to the construction in Section 3, however, the same solution $G$ is obtained from the Abel function based on the most regular behaviour as $z \rightarrow 1$. Considerations of this type are important in identifying the gamma function as the "best" solution of its functional equation (see, e.g., [4]); for other examples see Kneser [6], Szekeres [12].

In view of Equ. (12), pointing to a possibly complicated logarithmic singularity at $z=0$ we introduce

$$
\Theta(z):=\exp \Phi(z)
$$

satisfying the functional equation

$$
\Theta(1-z)=e^{p} \cdot \Theta\left(\sqrt{1-z^{2}}\right)
$$

which determines $\Theta(z)$ at most up to an arbitrary factor. The function $\Theta(z)$ satisfies an appropriate Schröder equation [10]. Assuming

$$
\Theta(z)=-\log (c z)+O(z)
$$

uniquely yields $p=\log 2, c=\frac{1}{2}$. In order to avoid logarithmic terms we attempt to find a formal solution $\Theta^{\prime}(z)=-z^{-1}+O(1)$ of the derivative of (23),

$$
\Theta^{\prime}(1-z)=\frac{2 z}{\sqrt{1-z^{2}}} \Theta^{\prime}\left(\sqrt{1-z^{2}}\right) \text {. }
$$

The procedure described below will directly result in a convergent series solving (24). Instead of $z$ we will use the variable $u:=\left(1-z^{2}\right) / 4 \in\left[0, \frac{1}{4}\right]$; therefore

$$
\sqrt{1-z^{2}}=2 \sqrt{u}, \quad z=\sqrt{1-4 u} \text {. }
$$

The function

$$
\vartheta(u):=-2 \Theta^{\prime}(2 u)
$$

then satisfies the functional equation

$$
\frac{1}{\sqrt{u}} \vartheta(\sqrt{u})=\frac{1}{\sqrt{1-4 u}} \vartheta\left(\frac{1-\sqrt{1-4 u}}{2}\right),
$$

for which a formal solution

$$
\vartheta(u)=c_{0} u^{-1}+c_{1} u+c_{2} u^{3}+c_{3} u^{5}+\ldots, c_{0}=1
$$

will be shown to exist. 
Lemma 4 For every $k \in \mathbb{R}$ the following expansion holds:

$$
f_{k}(u):=\frac{1}{\sqrt{1-4 u}}\left(\frac{1-\sqrt{1-4 u}}{2}\right)^{k}=\sum_{j=0}^{\infty}\left(\begin{array}{c}
k+2 j \\
j
\end{array}\right) u^{j+k}
$$

Proof: We only need the lemma for $k=-1,0,1 \ldots$. The correctness of the expansion is easily seen for $k=-1$ and $k=0$. Induction with respect to $k$ by using $f_{k+2}(u)-$ $f_{k+1}(u)+u f_{k}(u)=0$ and the basic relation between the elements of the Pascal triangle establishes Lemma 4 for $k=1,2,3, \ldots$.

Inserting the expansion (22) into (21) and using Lemma 4 directly yields the recurrence relation

$$
c_{k}=\sum_{j=0}^{\left[\frac{k}{2}\right]}\left(\begin{array}{c}
2 k-2 j-1 \\
k-1
\end{array}\right) c_{j}, \quad(k=0,1, \ldots), c_{0}=1
$$

for the sequence $c_{k}$. Its initial elements are

$$
c_{k}=\{1,1,4,13,49,181,685,2605,9988,38479,148879,577930,2249698, \ldots\} .
$$

Clearly, the coefficients $c_{k}$ form a monotonically increasing sequence of integers, and $c_{k} \leq 4^{k}$ may be shown. Therefore the series (28) converges for all $u \in \mathbb{C}$ with $|u|<\frac{1}{2}$, and from (26) we obtain

$$
\Theta(z)=-c_{0} \log \frac{z}{2}-\sum_{k=1}^{\infty} \frac{c_{k}}{2 k}\left(\frac{z}{2}\right)^{2 k}
$$

\section{Results}

In order to evaluate $\Theta(z)$ for a given $z \in(0,1)$, we use the functional equation (23) repeatedly before evaluating the series: Let $z_{0}:=z$ and iterate

$$
z_{j}=R\left(z_{j-1}\right)=\frac{z_{j-1}^{2}}{1+\sqrt{1-z_{j-1}^{2}}}, j=1,2, \ldots, m
$$

such that $\Theta\left(z_{m}\right)$ by the appropriately truncated series (31) has sufficient accuracy. Then $\Theta(z)=2^{-m} \Theta\left(z_{m}\right)$. The graphs of the functions $\Phi(z), \Theta(z)$ and $\exp (-\Theta(z))$ are shown in Figure $3 a$. 


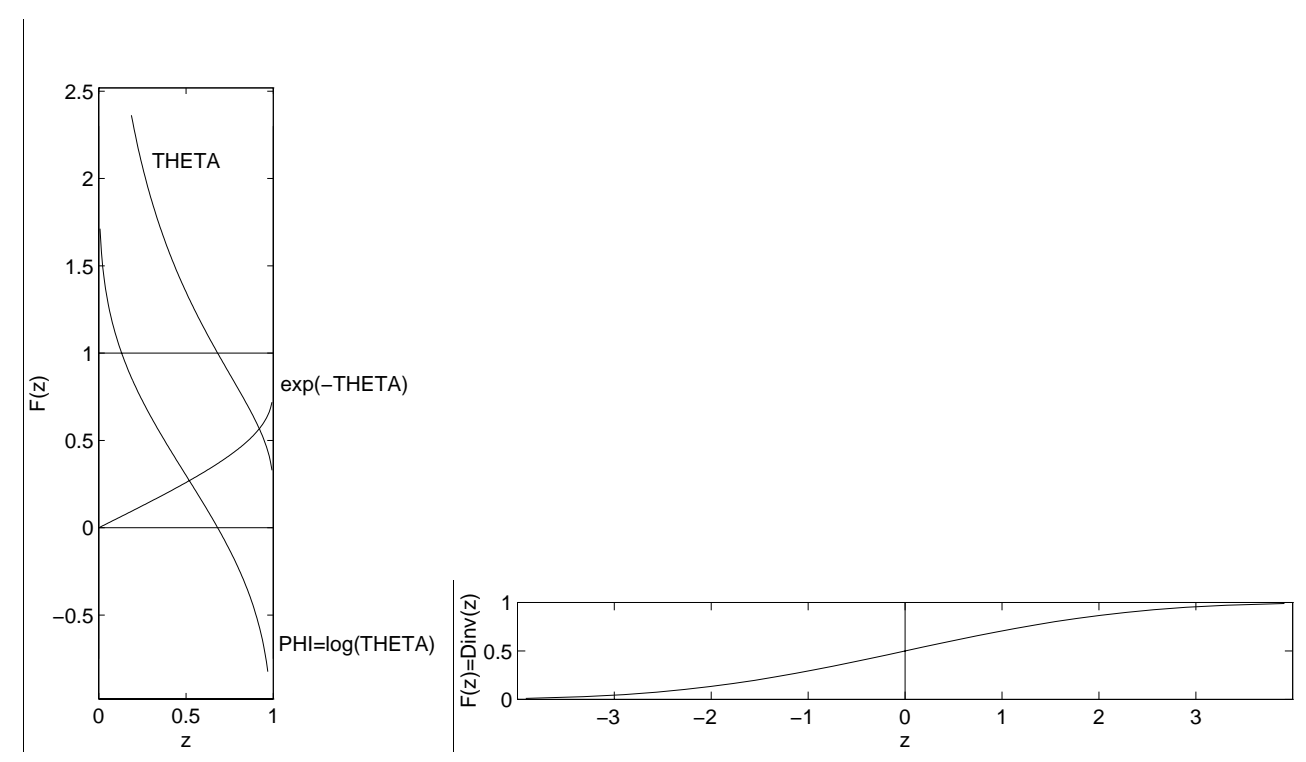

Fig. 3a: The functions $\Theta(z)$, $\exp (-\Theta(z))$, and $\Phi(z)=\log (\Theta(z)$ for $z \in(0,1)$

Fig. 3b: The inverse $F=D^{-1}$ of $D(z)=\log _{2}(\Theta(1-z) / \Theta(z))$

In terms of the Schröder function $\Theta$ the equation (15) of the graph $G(z)$ may be written as

$$
\Theta(1-G) \cdot \Theta(1-z)=2 \Theta(G) \cdot \Theta(z)
$$

as is seen by exponentiating Equ. (15). This is one form of the final result of the problem of Section 1 with $A$ and $B$ defined by Equ. (18). In order to solve (33) numerically for $G$ the secant method with the initial guesses $G_{0}=G_{0}(z):=\left(1-z^{\sqrt{2}}\right)^{1 / \sqrt{2}}$ and a nearby value $G_{1}$ is recommended.

Another representation of this graph is the parametrization (17) where, according to (16), the function $F(z)=D^{-1}(z)$ is now the inverse of

$$
D(z)=\log _{2} \frac{\Theta(1-z)}{\Theta(z)},
$$

to be computed, e.g., by solving the equation

$$
\Theta(1-F)=2^{z} \cdot \Theta(F)
$$

for the unknown $\mathrm{F}$ (see Figure $3 \mathrm{~b}$ ). As a consequence of (17), the fixed point $z_{0}=G\left(z_{0}\right)$ of the symmetric solution is now defined by $z_{0}=D^{-1}(1 / 2)$, which results in

$$
z_{0}=.606948137410748906864401661391988794557342957 .
$$

It is interesting to note that the solution $\widetilde{G}$ obtained directly from $\Theta$ by means of Equs (7), (9), (22), i.e.

$$
\widetilde{G}=A \Theta^{-1} \exp S_{\frac{p}{2}} \log \Theta \quad \text { or } \quad \tilde{G}(z)=1-\Theta^{-1}(\sqrt{2} \Theta(z)),
$$




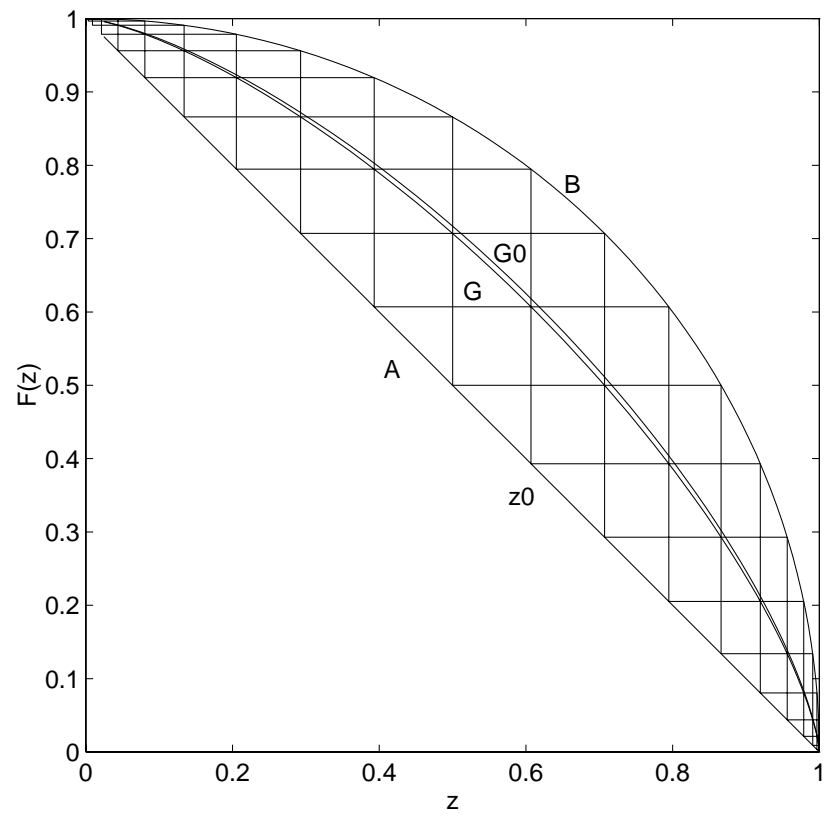

Fig. 4: Graphs of the functions $A(z), B(z), G(z), G_{0}(z)$ together with the four zigzagging polygons passing through the points $\left(\frac{1}{2}, \frac{1}{\sqrt{2}}\right)$, or $\left(z_{0}, z_{0}\right)$, respectively.

passes through the fixed point $z_{0}$ of $G$ and therefore through all of its forward and backward iterates under the map $A^{-1} B$. $\widetilde{G}$ is not quite symmetric, however; we have $|\tilde{G}(z)-G(z)| \leq 3.965894143 \cdot 10^{-7}$ and $|\widetilde{G}(\tilde{G}(z))-z| \leq 7.931788286 \cdot 10^{-7}$, see Figure 5 and Figure 6 . Since $\widetilde{G}(x)$ deviates from $G(z)$ only by an amount of less than $10^{-6}, \widetilde{G}$ is sufficient for graphics purposes.

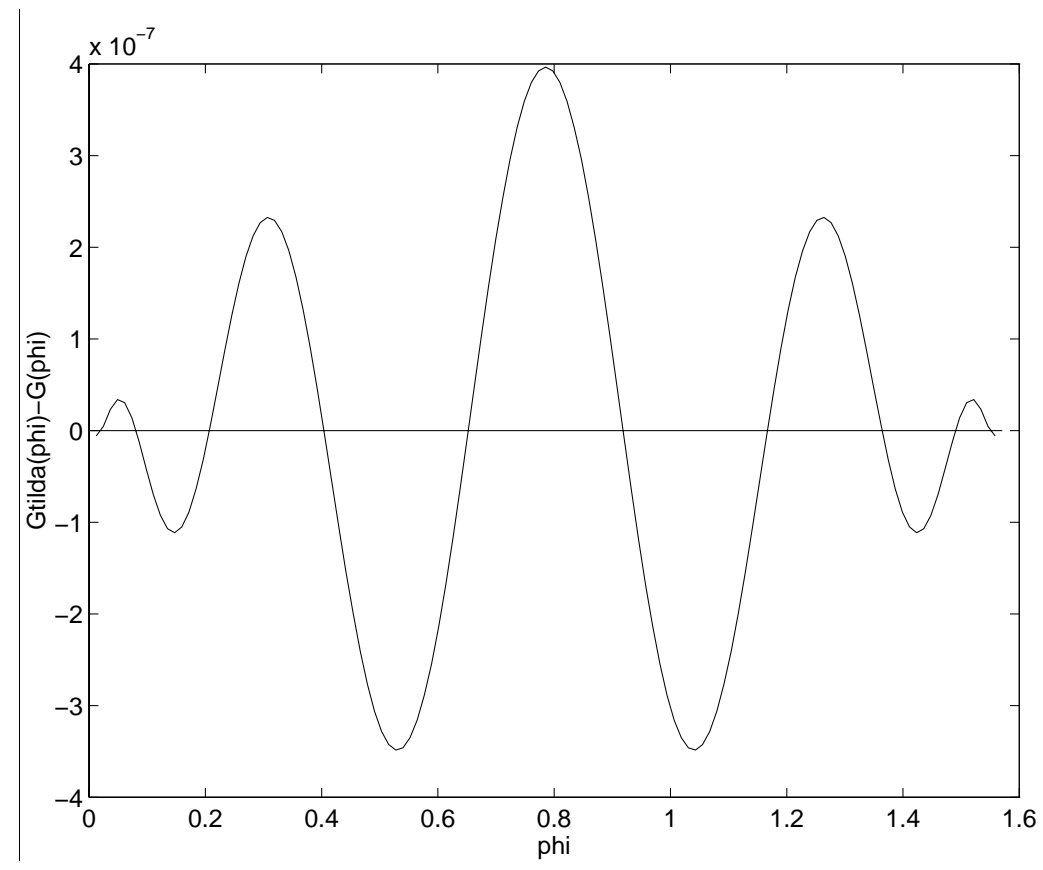

Fig. 5: Graph of the difference $\Delta(\varphi):=\widetilde{G}(\varphi)-G(\varphi)$, where $\cos (\varphi)=z$. This graph is not quite symmetric; we have $\left|\Delta(\varphi)-\Delta\left(\frac{\pi}{2}-\varphi\right)\right| \leq 1.156 \cdot 10^{-12}$. 


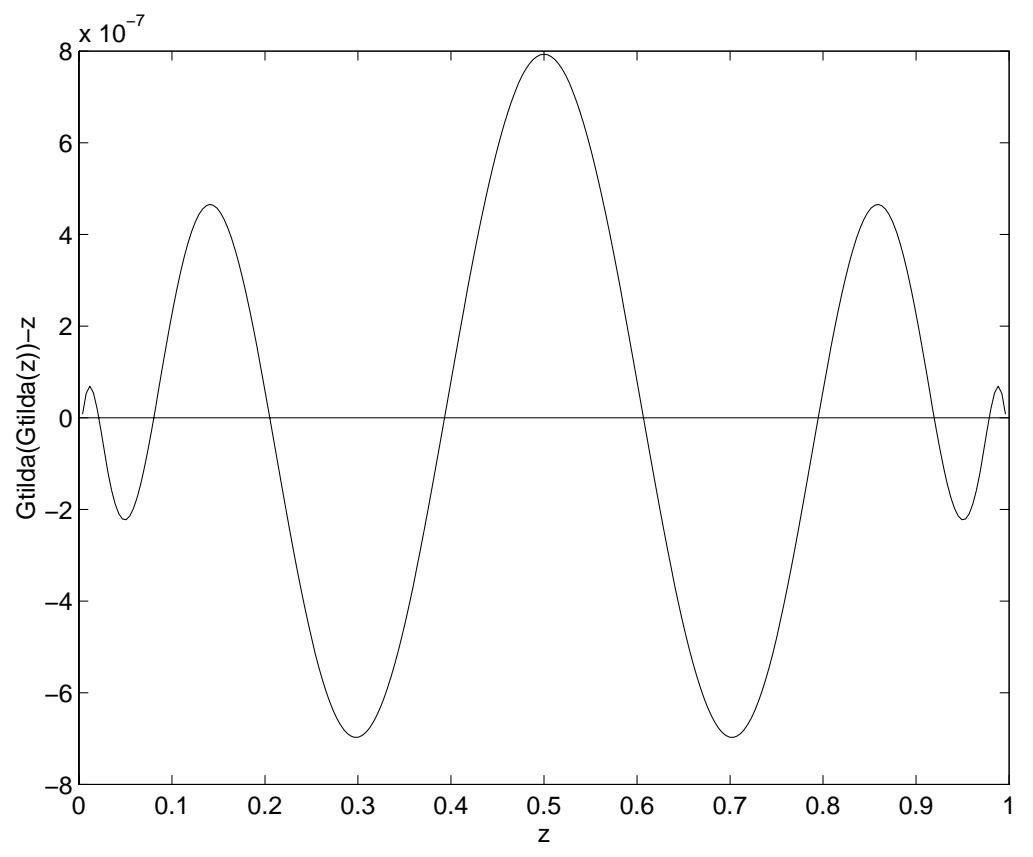

Fig. 6: Graph of the function $\tilde{G}(\tilde{G}(z))-z$

\section{Acknowledgments}

The authors are indebted to Philip J. Davis for his interest in the problem and for pointing out several relevant references.

\section{References}

[1] N. H. Abel, 1881: Détermination d'une fonction au moyen d'une équation qui ne contient qu'une seule variable. Euvres complètes, vol. 2, 36-39, Christiania (Oslo).

[2] L. Collatz, 1951: Numerische Behandlung von Differentialgleichungen. Springer, Berlin, 458 pp. In particular p. 419 ff.

[3] Ph. Davis, 1974: The Schwarz Function and its Applications. Carus Mathematical Monographs 17, Math. Assoc. of America, 228 pp.

[4] Ph. Davis, 1993: Spirals. From Theodorus to Chaos. With contributions by W. Gautschi and A. Iserles. A K Peters, Wellesley MA, 237 pp.

[5] E. Kasner, 1912: Conformal geometry. Proc. Fifth Int. Cong. Math., Cambridge, vol. 2, 81-87.

[6] H. Kneser, 1949: Reelle analytische Lösungen der Gleichung $\phi(\phi(x))=e^{x}$ und verwandten Funktionalgleichungen. J. reine angewandte Mathematik 187, 56-67. 
[7] G. Königs, 1884: Sur les intégrales de certaines équations fonctionelles. C. R. Acad. Sci. Paris 99, 1016-1017.

[8] M.Kuczma, 1968: Functional equations in a single variable. Monografie mat. 46. Polish Scientific Publishers, Warsaw.

[9] M.Kuczma, B. Choczewski, R. Ger, 1990: Iterative functional equations. Encyclopedia of Mathematics and its Applications 32. Cambridge University Press, 552 pp.

[10] E. Schröder, 1871: Über iterierte Funktionen. Math. Ann. 3, 296-322.

[11] F. Stenger, 1994: A Function Equation. NA Digest 10, item 2.

[12] G. Szekeres, 1962: Fractional itreation of exponentially growing functions. J. Australian Math. Soc. 2, 301-333.

[13] G.I. Targonski, 1964: A bibliography on functional equations. Research Report, Fordham University, Bronx N.Y., 96 pp.

[14] G.I. Targonski, 1981: Topics in iteration theory. Studia Mathematica Skript 6. Vandenhøeck \& Rupprecht, Göttingen, 292 pp. 